\title{
ACIDOSIS LACTICA SECUNDARIA A INTOXICACION POR METFORMINA, REPORTE DE CASO CLINICO.
}

Este artículo está bajo una icencia de Creative Commons de tipo Reconocimiento - No comercial - Sin obras derivadas 4.0 International.

OPEN ACCESS

1. Hospital "San Vicente de Paúl”. Médico Residente de la Unidad de Cuidados Intensivos. Ibarra - Ecuador 2. Hospital General Instituto Ecuatoriano de Seguridad Social. Médico Residente de la Unidad de Cuidados Intensivos. Ibarra - Ecuador.

\section{ORCID ID}

Ruiz Flores Andrés

orcid.org/0000-0001-6720-7653

Cevallos Pablo

orcid.org/0000-0001-7883-9533

*Corresponding author: Ruiz Flores Andrés. E-mail: ruizflores_1993@hotmail.com

\section{Article history}

Received: 15 - May - 2020

Accepted: 07 - Sep - 2020

Publish: 01 - Oct - 2020

CARE 2017 Check List statement: The authors have real the CARE 2017 Check List and the manuscript was prepared and revised according to the CARE 2017 Checklist.

\section{Conflict of interest: All authors declared that} there are no conflicts of interest.

Financial disclosure: The authors have no financial relationships relevant to this article to disclose

Authors' contribution: All the authors contributed in the search, selection of articles and writing. All the authors reviewed and approved the final manuscript.

Forma de citar este artículo: Ruiz Flores A, Pablo C. ACIDOSIS LACTICA SECUNDARIA A INTOXICACION POR METFORMINA, REPORTE DE CASO CLINICO. Rev Med Vozandes. 2020; 31 (2): $90-95$

\section{Resumen}

La metformina es un medicamento ampliamente utilizado como tratamiento de primera línea en diabetes mellitus tipo 2, su mecanismo de acción incluye impedir la elaboración de glucosa hepática, disminuye el transporte de glucosa a nivel intestinal entre otros. Uno de sus efectos adversos es la acidosis láctica, la mortalidad en estos pacientes aún es elevada a pesar de tratamientos modernos con terapia de sustitución renal.

Presentación del caso: Presentamos el caso de una paciente femenina de 16 años que ingresó al servicio de emergencia debido a intoxicación con metformina (30 tabletas de $500 \mathrm{mg}$ ) secundario a intento autolítico. La paciente se presentó somnolienta, con ruidos hidroaéreos aumentados, y relajación de esfínteres. Los controles gasométricos mostraron cifras de lactato en ascenso, llegando a valores de hasta $19,5 \mathrm{mmol} / \mathrm{L}$. Paciente requirió ventilación mecánica invasiva motivo por el cual, ingresó al servicio de Terapia Intensiva para manejo de fallo multiorgánico, se inició tratamiento sustitutivo renal con ciclos prolongados. Paciente fue dada de alta del servicio de terapia intensiva en su quinto día de hospitalización y continuó su tratamiento multidisciplinar a cargo del servicio de medicina interna.

Conclusiones: La metformina al ser un medicamento ampliamente usado a nivel mundial, todo servicio de emergencias debería estar preparado para identificar los casos de intoxicación por este fármaco. Dentro de las intervenciones se incluye: fluidoterapia, drogas vasoactivas, soporte ventilatorio invasivo, bicarbonato de sodio y terapia de reemplazo renal; todas ellas se brindaron a nuestra paciente con resultados exitosos.

Palabras clave: Metformina, acidosis láctica, terapia de sustitución renal.

\section{Abstract LACTIC ACIDOSIS SECONDARY TO METFORMIN
POISONING, CLINICAL CASE REPORT}

Metformin is a medicine used as first-line treatment in type 2 diabetes mellitus, its mechanism of action includes among others: preventing the production of hepatic glucose, decreases the transport of glucose at intestinal level. One of its adverse effects is lactic acidosis, especially when its used in autolytic attempts. Mortality in these patients is still high despite modern treatment with renal replacement therapy.

Case presentation: We present the case of a 16-year-old female patient who entered the emergency service due to metformin poisoning (30 tablets of $500 \mathrm{mg}$ ) secondary to autolytic attempt. The patient was drowsy, with increased hydroaereal noises, and relaxation of sphincters. Gasometric controls showed rising lactate figures, reaching values of up to $19.5 \mathrm{mmol} / \mathrm{L}$. Patient required invasive mechanical ventilation which is why, entered the intensive therapy service for management of multiorgan failure, renal replacement treatment was initiated with prolonged cycles. Patient was discharged from intensive care on her fifth day of hospitalization and continued her treatment in charge of the internal medicine service.

Conclusions: Metformin being a widely used medicine worldwide, every emergency service should be prepared to identify cases of this drug. Interventions include: fluid therapy, vasoactive drugs, invasive ventilatory support, sodium bicarbonate, and renal replacement therapy; All of them were provided to our patient with successful results.

Keywords: Metformin, lactic acidosis, renal replacement therapy. 


\section{Introducción}

La metformina es una biguanida, antidiabético oral utilizada como tratamiento de primera línea en distintos países para la diabetes mellitus tipo 2. La metformina inhibe la producción de glucosa hepática, disminuye el transporte de glucosa a nivel intestinal y aumenta la sensibilidad de la insulina en pacientes no insulinodependientes. Su uso en estos pacientes se asocia a menor mortalidad e menor incidencia de eventos cardiovasculares. Este antidiabético oral es ampliamente usado con excelente adherencia al tratamiento, sin embargo, una de sus complicaciones descritas como efecto adverso es la acidosis láctica, de esta complicación deben distinguirse dos posibles escenarios, el primero asociado al uso continuo de este medicamento; en una revisión de 11800 pacientes tratados con metformina durante 2 años solo 2 pacientes desarrollaron acidosis láctica, con una incidencia es de 3 a 9 casos por cada 100.000 pacientes tratados por año, en donde la lesión renal presente en pacientes diabéticos es el principal factor de riesgo, y el segundo escenario cuando la administración de este medicamento se usa en intentos autolíticos. El envenenamiento por metformina tiene una tasa de mortalidad elevada que oscila entre el $30 \%$ a 50\%; y en pacientes con un $\mathrm{pH}$ inferior de 6,9 su mortalidad se eleva hasta el 83\%, a pesar del tratamiento moderno (fluidoterapia, drogas vasoactivas, soporte ventilatorio invasivo, bicarbonato de sodio y terapia de reemplazo renal). ${ }^{(1-4)}$

\section{Acidosis láctica}

Se define hiperlactatemia cuando encontramos niveles de lactato sérico superiores de $2 \mathrm{mmol} / \mathrm{L}$, en cambio encontramos acidosis láctica con valores de lactato sérico superiores a $4 \mathrm{mmol} / \mathrm{L}$; la principal causa de acidosis metabólica es la acidosis láctica. ${ }^{(5,6)}$

La acidosis láctica se produce por un desequilibrio entre la producción y eliminación, normalmente producimos entre 15 a $20 \mathrm{mmol} / \mathrm{kg}$ por día, los mismos que son utilizados en el metabolismo de la glucosa, el equilibrio se logra mediante un consumo igual de ácido láctico.(5) Una posible razón mediante el cual la metformina causa acidosis láctica es bloqueando la piruvato carboxilasa, dando como efecto un aumento acelerado de lactato y metabolismo reducido. ${ }^{(2)}$

La acidosis láctica resulta la causa más primordial de toxicidad por metformina, aunque no se especifica la dosis tóxica(7)

La metformina favorece el cambio de glucosa en lactato en el lecho esplácnico intestino delgado, así mismo inhibe la síntesis de glucosa a partir de fuentes como lactato, piruvato y alanina dando como resultado el aumento de la productividad de lactato. ${ }^{(7)}$

El freno parcial del complejo de fosforilación oxidativa 1 de la cadena de transporte de electrones mitocondrial es el mecanismo más aceptado de acidosis láctica causado por metformina.

Así mismo se ha documentado la existencia de vasodilatación causando un colapso hemodinámico secundario al envenenamiento por metformina. ${ }^{(8)}$
La incidencia de toxicidad en pacientes que no tienen comorbilidades es infrecuente. Pero existe una mortalidad elevada asociada a sobredosis de metformina de raras circunstancias, que se asocia a las comorbilidades existentes en los pacientes ${ }^{(7)}$. Así mismo existe correlación entre niveles de lactato elevados y pH sérico bajo, se asocian a mayor mortalidad.(7)

\section{Presentación clínica}

La sintomatología es muy inespecífica, pero se ha demostrado en casos de sobredosis la presencia de náuseas y dolor abdominal. En otro escenario como es el caso de almacenamiento de acidosis láctica, clínica se caracteriza por molestias gastrointestinales tales como nauseas, vómito y diarrea, sucesivamente puede existir dificultad para respirar, hipotensión. A demás puede existir deterioro del estado neurológico secundario a hipoglicemia o acidosis. (7)

\section{Exámenes de gabinete}

Los exámenes iniciales incluyen(7):

1. Glicemia capilar para descartar hipoglicemia.

2. Niveles de otros tóxicos para descartar ingestiones concomitantes.

3. EKG para verificar trastornos en la conducción

4.Test de embarazo, en mujeres en edad reproductiva.

5. Gasometría arterial

6. Función renal y bicarbonato de sodio

7. Lactato sérico.

\section{Tratamiento}

\section{Vía área}

El estado neurológico del paciente además de la hiperpnea y taquipnea, respuestas fisiológicas compensatorios a la acidosis metabólica, deben ser consideradas antes de intubar al paciente, así como para establecer los parámetros ventilatorios. ${ }^{(7)}$

\section{Circulación}

La hipotensión requerirá inicialmente cristaloides, pero si no hay respuesta se debe continuar con vasoactivos. Cuando la hipotensión se mantiene, puede estar en relación a una toxicidad elevada, en donde se recomienda terapia de sustitución renal.(7)

\section{Gastrointestinal}

En pacientes con ingesta aguda, se indica lavado gastrointestinal con carbón activado, excepto si existen contraindicaciones específicas, así mismo se debe estimar el riesgo de broncoaspiración. La dosis es de $1 \mathrm{~g} / \mathrm{kg}$ (generalmente $50 \mathrm{~g}$ en adultos). No se debe administrar este tratamiento en pacientes 
con toxicidad crónica debido a que no se benefician del procedimiento. ${ }^{(7)}$

\section{Hipoglicemia}

No es común este signo clínico en la intoxicación solo por metformina, por lo cual se deben valorar otras posibles causas. Se indica soluciones glucosadas a 0,5 a $1 \mathrm{~g} / \mathrm{kg}$, seguido se puede administrar alimentos, si los mismos no están contraindicados. ${ }^{(7)}$

\section{Acidosis láctica}

\section{Bicarbonato de sodio}

La administración de bicarbonato de sodio presenta ciertos inconvenientes; desviación hacia la izquierda de la curva de disociación de hemoglobina, exceso de carga de sodio, alcalosis metabólica concomitante, variaciones en el potasio y calcio sérico, decrecimiento de la contractibilidad cardiaca, entre otros. A pesar de esto se recomienda la administración en pacientes con acidosis metabólica severa $(\mathrm{pH}$ arterial menor de 7.10 a 7.15) con la finalidad de mantener el pH superior a 7.15, hasta superar la toxicidad. ${ }^{(7)}$

\section{Hemodiálisis}

Existe mayor beneficio que riesgo en pacientes con acidosis láctica asociado intoxicación aguda de metformina; especialmente se indica la terapia de sustitución renal en los hallazgos mostrados en la tabla $1 .{ }^{(7,9)}$

Tabla 1. Hallazgos en donde se recomienda hemodiálisis.

\begin{tabular}{|c|c|}
\hline \multirow[t]{3}{*}{ Recomendación } & $\begin{array}{l}\text { Concentración sérica de lactato muy } \\
\text { elevada (> } 20 \mathrm{mmol} / \mathrm{L})\end{array}$ \\
\hline & Acidosis metabólica severa $(\mathrm{pH} \leq 7.0)$ \\
\hline & $\begin{array}{l}\text { Falta de mejora (según lo determinado } \\
\text { por el pH, la concentración de lactato } \\
\text { o el estado clínico) con atención de } \\
\text { apoyo y terapia con bicarbonato den- } \\
\text { tro de dos a cuatro horas }\end{array}$ \\
\hline \multirow[t]{5}{*}{ Evidencia limitada } & $\begin{array}{l}\text { Concentración elevada de lactato } \\
\text { sérico entre } 15 \text { y } 20 \mathrm{mmol} / \mathrm{L}\end{array}$ \\
\hline & Acidosis metabólica (pH de 7.0 a 7.1 ) \\
\hline & Comorbilidades: \\
\hline & $\begin{array}{l}\text { - Choque o inestabilidad hemodiná- } \\
\text { mica persistente que requiere terapia } \\
\text { vasopresora a pesar de la administra- } \\
\text { ción aguda de bolos IV de cristaloides } \\
\text { isotónicos por un total de } 30 \mathrm{ml} / \mathrm{kg}\end{array}$ \\
\hline & $\begin{array}{l}\text { - Lesión renal: creatina }>2 \mathrm{mg} / \mathrm{dL} \\
\text { (adultos), o> } 1.5 \mathrm{mg} / \mathrm{dL} \text { (ancianos), o } \\
2 \text { veces el límite superior de la enfer- } \\
\text { medad renal normal (niños) o crónica } \\
\text { (etapa } 3 \mathrm{~b} \text { o superior con TFGe < } 45 \mathrm{ml} / \\
\text { min / } 1.73 \mathrm{~m}^{2} \text {, oliguria o anuria) }\end{array}$ \\
\hline
\end{tabular}

Fuente: Jason Chu, MDAndrew Stolbach, MD, MPH, FAACT, FACMT F. Metformin poisoning [Internet]. UpToDate. 2020. Available from: https://www.uptodate.com/contents/metformin-poisoning/ print? search=acidosis lactic\&topic $R$ ef $=2370 \&$ source $=$ see_link

Es importante que el manejo de estos pacientes sea en conjunto con nefrología. Hasta el momento no se ha descrito un antídoto específico para la intoxicación por metformina.

Debido a su elevada mortalidad, baja incidencia y al no existir casos reportados a nivel nacional es importante hacer una revisión de la presentación clínica, diagnóstico y tratamiento; por lo cual a continuación presentamos el caso de una paciente adolescente que presentó intoxicación aguda por metformina secundario a intento autolítico en el Hospital San Vicente de Paúl de la ciudad de lbarra- Ecuador. Este manuscrito sigue las directrices CARE para el reporte de casos clínicos. ${ }^{(10)}$

\section{Presentacion del caso}

\section{Información del paciente}

Paciente femenina 16 años, estudiante, mestiza, sin antecedentes patológicos personales ni familiares de importancia clínica o quirúrgica, acudió a la emergencia del Hospital "San Vicente de Paúl" acompañada de su familiar por intento autolítico secundario a la administración de 30 tabletas de metformina 500 mg aproximadamente 2 horas 30 minutos antes de su arribo hospitalario.

\section{Hallazgos clínicos}

Los signos vitales se encontraron dentro de parámetros normales, al examen físico se encontraba somnolienta, con ruidos hidroaéreos aumentados y relajación de esfínteres.

Los exámenes de laboratorio en cifras anormales en varios parámetros (tabla 2) demostraron acidosis metabólica más acidosis láctica.

Con estos resultados se decidió intubación endotraqueal e interconsulta a nefrología para decidir la posibilidad de diálisis urgente, instaurándose terapia de sustitución renal en las horas siguientes. Durante la diálisis presentó hipotensión con TAM $55 \mathrm{mmHg}$ refractaria a la administración de cristaloides, se inició vasoactivo por vía periférica y se colocó catéter venoso central yugular interno izquierdo sin complicaciones. Con estas medidas se obtuvieron TAM de $60 \mathrm{mmHg}$ y continuó la terapia de sustitución renal. Los controles gasométricos posteriores evidenciaron acidosis metabólica severa (pH 7,1 y HCO3 8,2) que se corrigió con infusiones continuas de bicarbonato de sodio durante el procedimiento dialítico y posterior al mismo.

\section{Intervenciones terapéuticas}

La inestabilidad hemodinámica que presentó la paciente desde el inicio de la diálisis requirió 
Tabla 2. Exámenes de laboratorio.

\begin{tabular}{|c|c|c|c|c|c|c|c|}
\hline \multirow{18}{*}{ HEMOGRAMA } & TIPO DE ANÁLISIS (VALOR DE REFERENCIA) & Ingreso & Día 1 & Día 2 & Día 3 & Día 4 & Alta \\
\hline & LEUCOCITOS (4,50 - 11,30 103/mm3) & 6350 & 27,63 & 8,57 & 12,61 & 8,63 & 8,08 \\
\hline & HEMOGLOBINA $(12,0-15,4$ g/dl) & 12,8 & 13,4 & 10,3 & 10,3 & 8,6 & 7,9 \\
\hline & HEMATOCRITO (36.1- $47 \%$ mujeres) & 36 & 41,2 & 28,6 & 28,8 & 25,5 & 22,9 \\
\hline & PLAQUETAS (150 - $450 \quad$ 103/mm3) & 234 & 315 & 114 & 133 & 118 & 118 \\
\hline & NEUTRÓFILOS (55-65 \%) & 49 & 77 & 69 & 81 & 80 & 62 \\
\hline & GLUCOSA (70-100 mg/dl) & 173 & 250 & 266 & 168 & 126 & 89,7 \\
\hline & UREA (16-39 mg/dl) & 26 & 18 & 43 & 102 & 70 & 48,9 \\
\hline & CREATININA $(0,6-1,0 \mathrm{mg} / \mathrm{dl})$ & 1,06 & 1,19 & 1,46 & 2,24 & 1,3 & 0,91 \\
\hline & TGO (AST) $(0-41 \quad U / I)$ & 17 & 102 & 63 & 64 & 28 & 26 \\
\hline & TGP (ALT) (14-59 U/I) & 26 & 61 & 61 & 56 & 36 & 30 \\
\hline & BILIRRUBINA TOTAL $(0,10-1.0 \mathrm{mg} / \mathrm{dl})$ & 0,68 & & 0,63 & & 0,41 & 0,29 \\
\hline & BILIRRUBINA DIRECTA $(0,0-0,30 \mathrm{mg} / \mathrm{dl})$ & 0,23 & & 0,22 & & 0,16 & 0,12 \\
\hline & BILIRRUBINA INDIRECTA $(0,0-0,70 \mathrm{mg} / \mathrm{dl})$ & 0,46 & & 0,42 & & 0,25 & 0,17 \\
\hline & SODIO (135,0 - 145,0 mEq/l) & 131 & 133 & 136 & 142 & 144 & 141 \\
\hline & POTASIO (3.5 - $5.0 \mathrm{mEq} / \mathrm{l})$ & 4,05 & 4 & 3,16 & 3,93 & 4,28 & 3,89 \\
\hline & CLORO (95-1 $15 \mathrm{mEq} / \mathrm{l})$ & 102 & 83 & 95 & 105 & 112 & 109 \\
\hline & TEST DE EMBARAZO & Negativo & & & & & \\
\hline
\end{tabular}

Fuente: Elaborado por los autores

\section{GASOMETRIA ARTERIAL}

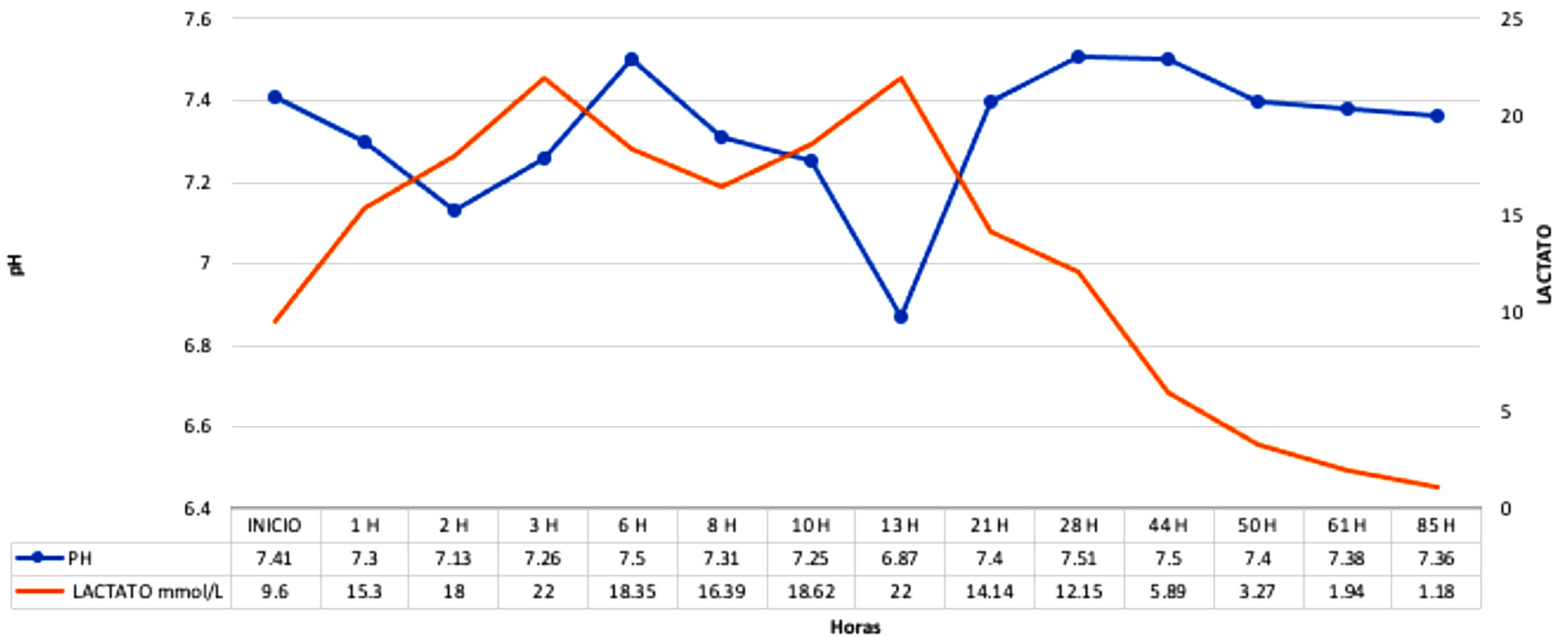

Gráfico 1. Gasometría arterial

dosis crecientes de norepinefrina para mantener TAM $\geq$ $60 \mathrm{mmHg}$. La taquicardia sinusal (135lpm) no se asoció a ninguna condición patológica.

La terapia de sustitución renal se realizó durante nueve horas continuas el primer día, al persistir alteraciones gasométricas, se decidió continuar con ciclos prolongados de diálisis durante le segundo día hasta corrección de parámetros gasométricos. Al tercer día se inició el destete de la ventilación mecánica, los controles gasométricos fueron adecuados, no se reportaron secreciones traqueales y fue extubada en la noche con buena mecánica ventilatoria y pulsioximetrías $>90 \%$.

Con el objetivo de disminuir el aporte vasocactivo, se optó por mejorar el compartimento intravascular con cristaloides y hemoderivados, con resultados adecuados. No fue necesario reintroducir terapia 
dialítica. Después de cinco días en UCl con evolución favorable, buen pronóstico, la paciente fue dada de alta para manejo multidisciplinario en el servicio de Medicina Interna.

\section{Discusión}

La metformina, una biguanida, es un fármaco perteneciente al grupo de los antidiabéticos orales ampliamente usado solo o combinado en pacientes con diabetes mellitus tipo $2^{(11)}$, es una molécula pequeña de 165 Da, que tiene una biodisponibilidad oral del $55 \%$ y un volumen de distribución de $1-5 \mathrm{l} / \mathrm{kg}$. Su excreción se da sin cambios vía renal, su aclaramiento corporal total en sujetos con función renal conservada es de $500 \mathrm{ml} / \mathrm{min}$, aquellos con diálisis intermitente es de $200 \mathrm{ml} / \mathrm{min}$ y $50 \mathrm{ml} / \mathrm{min}$ en pacientes con Terapia de Reemplazo Renal Lento Continua (TRRLC). Las concentraciones terapéuticas son de 1,5-3,0mg/l. La semivida de eliminación en personas sin disfunción renal es de 8 a 20 horas. ${ }^{(12)}$

Más allá de su efecto en el metabolismo de la glucosa, se ha encontrado otros beneficios entre ellos: restaurar la función ovárica en el Síndrome de Ovario Poliquístico (SOPQ), su Uso en hígado graso, para minimizar complicaciones macro y microvasculares de la diabetes mellitus tipo 2; y últimamente se encuentra en estudio como adyuvante en terapias oncológicas y para diabetes gestacional; así como prevención en poblaciones pre-diabéticas. ${ }^{(11)}$ Debido a que cada vez se amplía su uso en diferentes situaciones clínicas y en distintos grupos etarios, se aumenta el riesgo de que puedan suceder efectos adversos graves por este fármaco. Sus efectos secundarios más frecuentes son leves y son de tipo gastrointestinal (nausea, vómito y diarrea); sin embargo, cuando sobrepasamos las concentraciones terapéuticas puede ocurrir acidosis láctica, su efecto adverso más temido. ${ }^{(13)}$

En cuanto al manejo de la acidosis láctica, a pesar que por largo tiempo y grandes guías internacionales recomiendan el uso de bicarbonato de sodio $\left(\mathrm{NaHCO}_{3}\right)$ en el manejo de la acidosis con el fin de mejorar la hemodinamía y disminuir la cantidad de vasopresores en la acidosis láctica secundaria a hipoperfusión (tipo A) hasta que se instaura el tratamiento definitivo (14); en el caso de la acidosis láctica no causada por hipoperfusión (tipo B) su uso de rutina sigue siendo controversial debido a que podría producir cambios en la curva de disociación de la hemoglobina, aumento de la producción de $\mathrm{CO}_{2}$, aporte extra de sodio, empeoramiento de la acidosis intracelular, disminución del Ca+ sérico que se traduce en disminución del tono vascular y la contractilidad cardíaca ${ }^{(12,15)}$, razón por lo que los pacientes en su mayoría se encuentran hipotensos, ante lo cual su manejo es inicialmente con soluciones cristaloides, seguido de drogas vasoactivas en caso de ser necesario (3) Sin embargo tanto como la literatura reporta, así como en el caso presentado, se puede considerar la terapia con bicarbonato de sodio para prevenir un futuro empeoramiento en el equilibrio acido base hasta instaurar las medidas de reemplazo renal y soporte de fallo multiorgánico, en nuestra paciente no se reportó efectos adversos y se obtuvo buen resultado. En este tipo de intoxicaciones la terapia extracorpórea con hemodiálisis es el tratamiento de primera elección en pacientes con estabilidad hemodinámica, y en aquellos hemodinamicamente inestables se prefiere usar TRRLC a pesar de su evidencia limitada.
ElGrupo deTrabajo deTratamientosExtracorpóreos en Envenenamientos (Extracorporeal Treatments In Poisoning Workgroup) concluye que la metformina es una molécula moderadamente dializable por lo que el tratamiento extracorpóreo es recomendado en intoxicación severa y emite recomendaciones. Entre ellas habla sobre las indicaciones para terapia extracorpórea: lactato $>20 \mathrm{mmol} / \mathrm{L} ; \mathrm{pH}<$ o igual a 7.0, shock, fallo de las medidas estándar de soporte vital y alteración en el nivel de conciencia. Recomiendan que debe darse tratamiento extracorpóreo hasta que el lactato sérico sea $<3 \mathrm{mmol} / \mathrm{L}$ y $\mathrm{pH}>7.35$. Sobre qué tipo se prefiere, recomiendan hemodiálisis como primera elección, si no está disponible se deben considerar terapias continuas de reemplazo renal. (17) Nuestra paciente fue sometida a hemodiálisis convencional extendida con resultados favorables en el día 1, durante 9 horas, con esto logró descender su lactato sérico a $3.27 \mathrm{mmol} / \mathrm{L}$ para su segundo día, sin necesidad de repetir la terapia dialítica.

La evolución de la paciente presentada fue favorable después de las intervenciones del día 1, se tuvo excelente mejoría en cifras gasométricas y de lactato luego de la terapia de sustitución renal, se retiró la ventilación mecánica al día 2 y el soporte vasoactivo al día 4. Paciente en quien no se observa exacerbación de los fallos orgánicos previos en las siguientes 24 horas por lo cual se decide el alta de la Unidad de Cuidados Intensivos para su posterior manejo por en hospitalización del servicio de Medicina Interna.

\section{Conclusiones}

La acidosis láctica inducida por metformina es un escenario que a pesar de no ser tan frecuente como otro tipo de intoxicaciones o envenenamientos, siempre puede presentarse al servicio de emergencias por lo que se debe distinguir el tipo de paciente que del que se trata (intento autolítico vs intoxicación en paciente con factores de riesgo), esto junto con la gravedad inicial nos conducirá a tomar las medidas terapéuticas: fluidoterapia, drogas vasoactivas, soporte ventilatorio invasivo, bicarbonato de sodio y terapia de reemplazo renal; cómo ya se mencionó, la terapia extracorpórea es la intervención que más reduce la mortalidad en estos pacientes, de la cual se prefiere hemodiálisis en paciente con estabilidad hemodinámica, y terapia de reemplazo renal lento continúa en quienes no se ha alcanzado adecuada hemodinamía. 


\section{Referencias}

1. Leonaviciute D, Madsen B, Schmedes A, Buus $\mathrm{NH}$, Rasmussen BS. Severe Metformin Poisoning Successfully Treated with Simultaneou Venovenous Hemofiltration and Prolonged Intermittent Hemodialysis. Case Reports Crit Care. 2018;2018:1-4.

2. van Berlo-van de Laar IRF, Vermeij CG, van den Elsen-Hutten M, de Meijer A, Taxis K, Jansman FGA. Extracorporeal treatment of metforminassociated lactic acidosis in clinica practice: a retrospective cohort study. Eur Clin Pharmacol. 2020;

3. Sánchez-Díaz JS, Monares-Zepeda E, Martínez-Rodríguez EA, Cortés-Román JS, TorresAguilar O, Peniche-Moguel KG, et al. Acidosis láctica por metformina: reporte de caso. Rev Colomb Anestesiol. 2017;45(4):353-9.

4. Defronzo R, Fleming GA, Chen K, Bicsak TA Metformin-associated lactic acidosis: Current perspectives on causes and risk. Metabolism. 2016;65(2):20-9.

5. Michael Emmett, MDHarold Szerlip, MD, FACP FCCP, FASN F. Causes of lactic acidosis. UpToDate. 2020.

6. Morris CG, Low J. Metabolic acidosis in the critically ill: Part 2. Causes and treatment. Anaesthesia. 2008;63(4):396-411.
7. Jason Chu, MDAndrew Stolbach, MD, MPH, FAACT, FACMT F. Metformin poisoning. UpToDate. 2020.

8. DONALD W. LANDRY MD. PD AND JUAN A. OLIVER MD. THE PATHOGENESIS OF VASODILATORY SHOCK. N Engl J Med. 2001;345(8):58895

9. Harbord N. Common Toxidromes and the Role of Extracorporeal Detoxification. Adv Chronic Kidney Dis. 2020;27(1):1 1-7.

10. Gagnier J, Kienle G, Altman D, Moher D, Sox $H$, Riley D. The CARE guidelines: consensusbased clinical case reporting guideline development. Journal of Medical Case Reports. 2013;7(1).

11. Viollet B, Guigas B, Sanz Garcia N, Leclerc J, Foretz $M$, Andreelli $F$. Cellular and molecular mechanisms of metformin: An overview. Clin mechanisms of metformir
Sci. 2012;122(6):253-70.

12. Graham GG, Punt J, Arora M, Day RO, Doogue MP, Duong JK, et al. Clinical pharmacokinetics of metformin. Clin Pharmacokinet. $2011 ; 50(2): 81-98$.

13. Friesecke $S$, Abel $P$, Roser $M$, Felix SB, Runge $S$. Outcome of severe lactic acidosis associated with metformin accumulation. Crit Care. 2010;14(6)
14. Dellinger RP, Levy $M$, Rhodes $A$, Annane $D$ Gerlach H, Opal SM, et al. Surviving sepsis campaign: International guidelines for management of severe sepsis and septic shock: 2012. Crit Care Med. 2013:41 (2):580-637.

15. Velissaris D, Karamouzos $\vee$, Ktenopoulos $N$, Pierrakos C, Karanikolas M. The use of sodium bicarbonate in the treatment of acidosis in sepsis: A literature update on a long term debate. Crit Care Res Pract. 2015;2015(March)

16. Levraut J, Giunti C, Ciebiera JP, De Sousa $G$, Ramhani $R$, Payan $P$, et al. Initial effect of sodium bicarbonate on intracellular $\mathrm{pH}$ depends on the extracellular nonbicarbonate buffering capacity. Crit Care Med. 2001;29(5):1033-9.

17. Calello DP, Liu KD, Wiegand TJ, Roberts DM Lavergne V, Gosselin S, et al. Extracorporeal treatment for metformin poisoning: Systema-
tic review and recommendations from the tic review and recommendations from the
extracorporeal treatments in poisoning workgroup. Crit Care Med. 2015;43(8):1716-30. 